\title{
Laser Deceleration of an Atomic Beam
}

\author{
William D. Phillips and Harold Metcalf ${ }^{(a)}$ \\ Electrical Measurements and Standards Division Center for Absolute Physical Quantities, \\ National Bureau of Standards, Washington, D. C. 20234
}

(Received 23 December 1981)

\begin{abstract}
Deceleration and velocity bunching of $\mathrm{Na}$ atoms in an atomic beam have been observed. The deceleration, caused by absorption of counterpropagating resonant laser light, amounts to $40 \%$ of the initial thermal velocity, corresponding to about 15000 absorptions. Atoms were kept in resonance with the laser by using a spatially varying magnetic field to provide a changing Zeeman shift to compensate for the changing Doppler shift as the atoms decelerated.
\end{abstract}

PACS numbers: $32.90 .+\mathrm{a}, 32.30 . \mathrm{Bv}$

We report a definitive observation of the deceleration and velocity bunching of neutral atoms in a thermal beam by momentum transfer from a counterpropagating resonant laser beam. $\mathrm{Al}$ though ions trapped by electromagnetic fields have been cooled by laser light, ${ }^{1}$ such traps are not deep enough to confine thermal neutral atoms for similar cooling. A sample of slow neutral atoms could alleviate problems such as transit time and second-order Doppler effects in atomic clocks and precision spectroscopy. They may also be used for very-low-energy scattering or be deposited into shallow traps for further cooling.

Frisch first reported the deflection of atoms by resonant light in $1933,{ }^{2}$ but the availability of tunable lasers has recently stimulated new interest in controlling the motion of atoms with light. Cooling $^{3}$ and trapping ${ }^{4}$ of atoms with lasers have been proposed, and deflection, ${ }^{5}$ and steering and focusing, ${ }^{6}$ of atomic beams with lasers have been reported.

Although observable deflection can be accomplished by scattering a small number of photons, transfer of the momentum of a large number of photons is required for the thermal atoms to experience a significant change of momentum along the beam direction. The velocity change caused by scattering $n$ photons resonant with the $3 S$ to $3 P$ transition in Na (yellow $D$ line) is $n h \nu / M c$ $\simeq 3 n \mathrm{~cm} / \mathrm{s}$. When the transition is saturated by resonant light of intensity large compared with $10 \mathrm{~mW} / \mathrm{cm}^{2}$, so that the atom spends half its time in the $3 P$ state (lifetime $\simeq 16 \mathrm{~ns}$ ), the $\max$ imum deceleration of $a=(3 \mathrm{~cm} / \mathrm{s}) /(32 \mathrm{~ns}) \simeq 10^{8}$ $\mathrm{cm} / \mathrm{s}^{2}$ is achieved. Then atoms with $v_{0}=10^{5} \mathrm{~cm} / \mathrm{s}$ are stopped in about $1 \mathrm{~ms}$ over a $50-\mathrm{cm}$ path having scattered about 30000 photons.

Two processes limit deceleration by this method: Atoms experience a Doppler shift out of reso- nance with the laser as they slow down, and atoms are optically pumped. Optical pumping occurs when atoms are excited from one of the two Na hyperfine ground states (HFGS) to a $3 P$ state that decays to the other HFGS. Since these HFGS are separated by $1.77 \mathrm{GHz}$ and the natural linewidth is $10 \mathrm{MHz}$, atoms pumped to this other HFGS are not resonant with the laser. Both the Doppler shift and the optical pumping problems can be solved by using a spatially varying magnetic field applied along the laser-atomic-beam axis. The field both Zeeman tunes the decelerating atoms into constant resonance with the fixedfrequency cooling laser and produces selection rules and Zeeman shifts that strongly discriminate against optical pumping.

We use a circularly polarized cooling laser tuned to induce the $\sigma^{+}$transition from the $F=2$, $M_{F}=2$ sublevel of the $3 S_{1 / 2}$ ground state to the $F$ $=3, M_{F}=3$ sublevel of the $3 P_{3 / 2}$ excited state (neither of these states is mixed by the magnetic field). In this case the selection rules demand that transitions occur only between this particular pair of states with maximum $M_{F \cdot}$. Imperfect polarization, angular divergence, or misalignment of the laser and magnetic field axes allow transitions to other than the $F=M_{F}=3$ excited state. Nevertheless, if the magnetic field is at least $0.04 \mathrm{~T}$, either the matrix elements for unwanted excitations are so small or those transitions are Zeeman shifted so far out of resonance that little optical pumping results. For example, the probability of the $\pi$ transition from the $F=M_{F}$ $=2$ ground state to the $F=3, M_{F}=2$ excited state, much weaker than the $\sigma^{+}$transitions at zero field, is further reduced by a factor of 10 at $0.02 \mathrm{~T}$ and a factor of 100 at $0.05 \mathrm{~T}$. Also, the detuning for the allowed $\pi$ transition to the $F=2, M_{F}=2$ state is $400 \mathrm{MHz}$ at $0.02 \mathrm{~T}$ and almost $1000 \mathrm{MHz}$ at $0.05 \mathrm{~T}$. We estimate that the combined effect of 
alignment errors and finite magnetic field allows undesired transitions to be $10^{-4}$ times as probable as the desired $\sigma^{+}$transition.

Because the Zeeman shift $\gamma_{z}$ of the frequency of the $\sigma^{+}$transition is a constant $(14 \mathrm{GHz} / \mathrm{T})$, the changing Doppler shift of an atom experiencing uniform deceleration $a$ can be cancelled by a magnetic field varying as $B=B_{b}+B_{0}(1-2 a z)$ $\left.v_{0}^{2}\right)^{1 / 2}$. For $B_{0} \simeq 0.12 \mathrm{~T}$, the Zeeman shift matches the Doppler shift of atoms from velocity $v_{0}$ $=10^{5} \mathrm{~cm} / \mathrm{s}$ down to zero. Slower atoms begin decelerating upon reaching the magnetic field whose Zeeman shift matches their Doppler shift. Thus all atoms of velocity $v_{0} \leqslant \lambda \gamma_{\mathrm{Z}} B_{0}$ are bunched into one slow velocity group. With a constant bias field $B_{b}$ of at least $0.04 \mathrm{~T}$, the field described above solves both the Doppler-shift and optical-pumping problems.

This use of a changing magnetic field to follow the changing atomic Doppler shift "adiabatically" is similar in principle to the use of a rapidly changing laser frequency proposed by Letokhov ${ }^{7}$ to follow the changing Doppler shift. Balykin, Letokhov, and Mishin ${ }^{8}$ have reported deceleration of a $\mathrm{Na}$ beam with this technique, using the same frequency-scanned laser simultaneously to produce velocity analysis of the atomic beam. This procedure produces ambiguous results because the velocity distribution is inferred from the Doppler line shape which is distorted by optical pumping. ${ }^{9}$ Our procedure avoids these problems by using a second, weak laser to perform the velocity analysis. Furthermore, the use of a magnetic field to achieve adiabatic cooling results in a continuous, rather than a pulsed, production of slow atoms.

Our apparatus is shown schematically in Fig. 1. A $500{ }^{\circ} \mathrm{C}$ oven with a $600{ }^{\circ} \mathrm{C}$ nozzle produces a slightly supersonic $\mathrm{Na}$ beam that is then collimated to $\sim 0.01 \mathrm{rad}$. About $50 \mathrm{~cm}$ from the source the beam enters a $60-\mathrm{cm}$-long solenoid. Three layers of wire wound on the entire $60-\mathrm{cm}$ length provide the bias field $B_{b}$, while additional windings on only part of the solenoid produce the spatially varying part of the field.

The $30-\mathrm{mW}$ cooling laser is aligned so that its 5 -mm-diam beam overlaps the atomic beam for the entire 1.6-m length of the apparatus; the 80$\mu \mathrm{W}$ analyzing beam crosses these two at $\sim 6^{\circ}$, overlapping them in a region a few centimeters long in the field of view of a photomultiplier (PMT). (The laser linewidths are about $10 \mathrm{MHz}$.) This region is far enough away from the $9-\mathrm{cm}-$ diam solenoid to be in nearly zero field. The output of the PMT is amplified and fed to a boxcar integrator triggered by the $100-\mathrm{Hz}$ chopper interrupting the cooling beam.

The boxcar's dual 50- $\mu$ s gates are set to observe fluorescence induced by the analyzing laser after the cooling beam has been blocked by the chopper (fall time $\sim 15 \mu \mathrm{s}$ ). Gate $A$ is set $50 \mu \mathrm{s}$ after the cooling beam turns off and gate $B$ is set $4 \mathrm{~ms}$ later. The $A$ signal therefore comes from atoms that have traversed the solenoid while the cooling light was on (drifting only a few centimeters during the 50- $\mu$ s delay) while the $B$ signal comes from atoms that were never in the cooling laser light.

As the analyzing laser's frequency is scanned slowly, the observed fluorescence gives the ve-

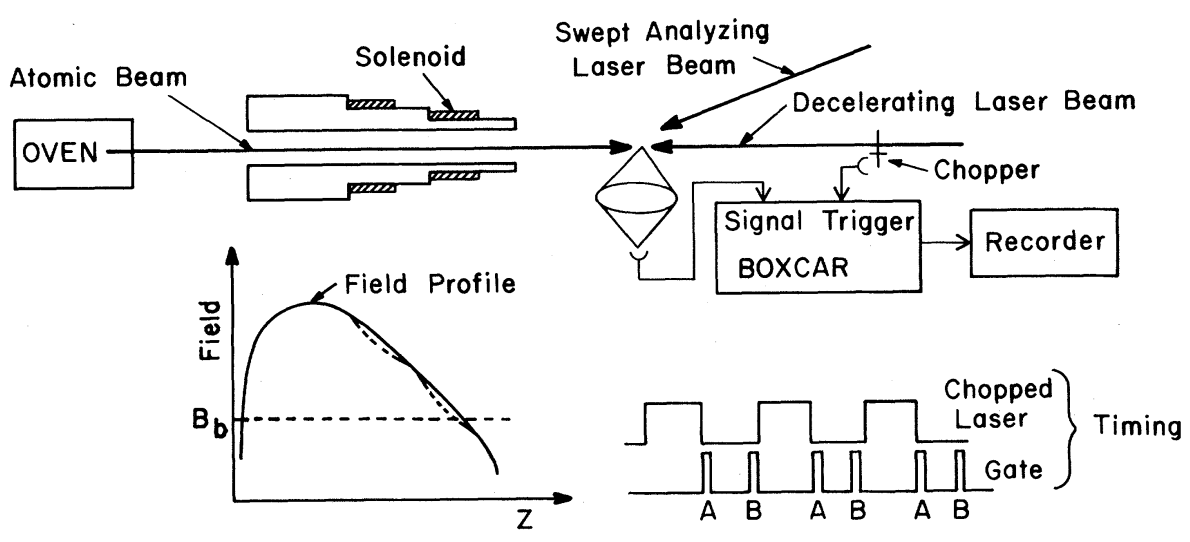

FIG. 1. Schematic diagram of apparatus including optical and electronic arrangement. A plot of $B$ vs $z$, with and without the turns in the small shaded area of the solenoid, is shown. Note the regions of large $d B / d z$ (steep slope on broken curve). The timing of the boxcar gates $A$ and $B$ relative to the chopping of the decelerating laser is shown at the lower right-hand side. 


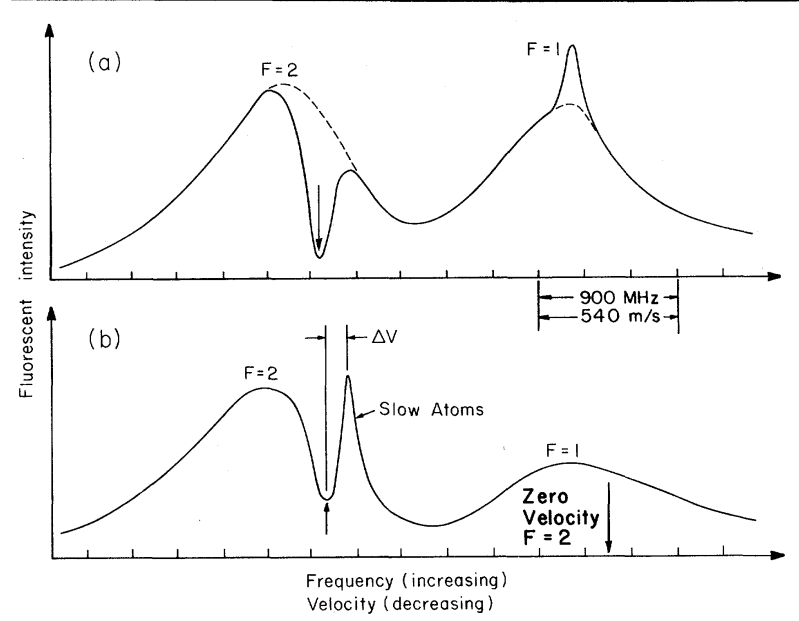

FIG. 2. (a) A scan of the probe laser with no field and highly attenuated deceleration laser shows the velocity distribution and hyperfine peaks ( $B$ trace, dashed line) and the optical pumping of $F=2$ atoms into the $F=1$ state ( $A$ trace, solid line). (b) A uniform 0.075-T field with the laser at full power results in a deep, broad optical-pumping hole for $M_{F} \neq 2, F=2$ atoms and a narrow, slow-speed peak in the middle of it produced by nonadiabatic deceleration. Tic marks are from a $300-\mathrm{MHz}$ free spectral range etalon. Arrows indicate the velocity group resonant with the cooling laser.

locity distribution of the atoms in the beam, higher frequency corresponding to lower velocity because of the Doppler shift. The $A$ signal gives the velocity distribution as modified by the cooling laser, while the $B$ signal gives the unmodified velocity distribution. The signal is complicated by the 1.77-GHz hfs splitting of the ground state that results in two peaks, separated by the hfs and showing the velocity distribution of atoms in the $F=1$ and $F=2$ HFGS. The $15-60-\mathrm{MHz}$ hfs splitting of the $\boldsymbol{P}_{3 / 2}$ state is unresolved. When the probe laser is weak the two peaks have similar shapes, but are scaled by the $3: 5$ ratio of populations in the two ground states.

Figure 2(a) shows the $A$ signal obtained when the cooling laser is attenuated by a factor of $10^{4}$ to about $3 \mu \mathrm{W}$, tuned to be in resonance with $F$ $=2$ ground-state atoms, and no magnetic field is applied. The $F=2$ atoms are pumped to $F=1$ producing a hole in the $F=2$ velocity distribution and a corresponding peak in the $F=1$ distribution. At higher power the hole and peak are made broader and stronger. Figure 2(b) shows the effect of applying only a uniform bias field of $0.075 \mathrm{~T}$, raising the laser to full power, and retuning it to compensate for the Zeeman shift. Atoms in the field are not optically pumped out

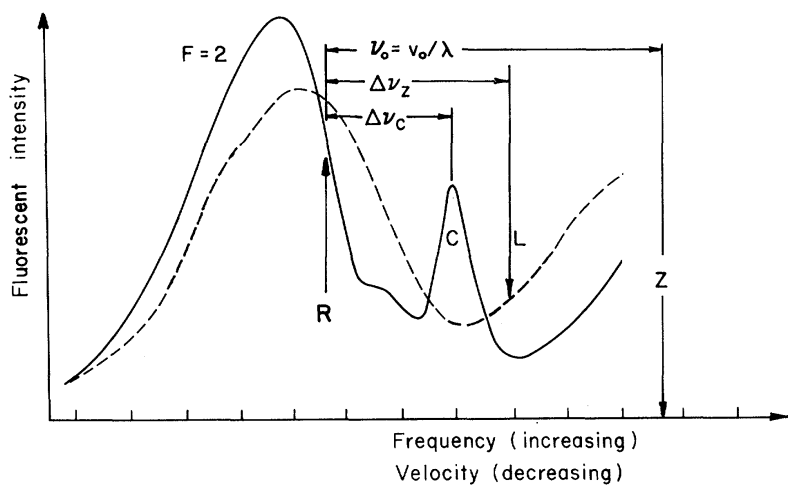

FIG. 3. Solid curve taken at full laser power with tapered field (maximum $\cong 0.06 \mathrm{~T}$ ). Peak $C$ is fluorescence from slow $F=2$ atoms. The arrow $R$ indicates the velocity group resonant with the cooling laser at maximum field Zeeman shifted by $\Delta \nu_{Z}, L$ indicates the resonant velocity group at zero field, and $Z$ indicates zero velocity for $F=2$ atoms. The quantity $\Delta \nu_{c}$ is the Doppler shift corresponding to the deceleration. The dashed curve is a $B$ trace clearly showing the absence of atoms with velocities near that of peak $C$. Tic marks are from a $300-\mathrm{MHz}$ free spectral range etalon.

of the $F=2$ ground state, and so repeated absorptions occur. This results in a hole in the $F=2$ velocity distribution, but there is no corresponding peak in the $F=1$ distribution. Therefore, the hole is not the result of optical pumping. A peak does occur, however, just on the low-velocity side of the hole because many atoms are transferred to a slower velocity group by repeated absorptions. The velocity change is about $15 \%$ of the initial velocity and is limited by the Doppler shifting of the cooled atoms out of resonance with the cooling laser. Minogin ${ }^{10}$ has analyzed this nonadiabatic cooling problem theoretically, and our results are in qualitative agreement.

To achieve adiabatic cooling, we connected the additional windings on the solenoid to produce a spatially varying magnetic field. The results are shown by the solid curve of Fig. 3. The arrow $R$ indicates the velocity group resonant with the cooling laser at the peak value of magnetic field $(0.06 \mathrm{~T})$. The arrow $L$ indicates the velocity group resonant at zero field [determined by optical pumping as in Fig. 2(a)] and is separated from $R$ by the Zeeman shift $\Delta \nu_{\mathrm{Z}}=0.06 \gamma_{\mathrm{Z}}$ $=840 \mathrm{MHz}$. Atoms slower than $v_{0}$ are decelerated and bunched into the highly populated velocity group at peak $C$ (much higher than the uncooled distribution shown by the dashed $B$ trace).

In order to determine the magnitude of $v_{0}$ 
(Doppler shift $=\nu_{0}$ ) a small part of the analysis laser beam was directed perpendicular to the atomic beam so that there is no first-order Doppler shift. The arrow $Z$ in Fig. 3 indicates the frequency at which fluorescence is induced by this laser beam and therefore marks the resonant frequency of zero-velocity $F=2$ atoms in zero field. The distance from $Z$ to the arrow $R$ gives $v_{0}=1180 \mathrm{~m} / \mathrm{s}$. The observed velocity change $\Delta v_{c}$ is $\simeq 40 \%$ of $v_{0}$ and corresponds to about 15000 absorptions.

The maximum deceleration we can achieve is now limited by the total change in the magnetic field. A larger field change could result in fur ther deceleration but the adiabatic requirement allows a certain maximum gradient. Since the maximum deceleration is $a=10^{8} \mathrm{~cm} / \mathrm{s}^{2}$, this maximum $d B / d z=a / \gamma_{Z} \lambda v$ where the $\lambda$ is from the Doppler shift and $\gamma_{\mathrm{z}}=14 \mathrm{MHz} / \mathrm{T}$. For $v \simeq 10^{5}$ $\mathrm{cm} / \mathrm{s},(d B / d z)_{\max }$ is $1 \mathrm{mT} / \mathrm{cm}(10 \mathrm{G} / \mathrm{cm})$. Our solenoid is already at this limit.

We tested this analysis of the limitation on cooling by disconnecting those turns on the solenoid indicated by the shaded areas in Fig. 1. This produced two regions where $d B / d z$ was too large (steep part of broken curve), with little change in the field between these regions. We found that atoms could not decelerate adiabatically from one end of the solenoid to the other.

A larger change in the magnetic field, over a longer path, will be needed to achieve greater cooling. We believe that we can reduce the atomic velocity by at least a factor of 10 from thermal velocity before transverse heating effects, ${ }^{11,12}$ caused by the random reemission, and increased angular divergence become important problems. This work was supported in part by the U. S. Office of Naval Research.

\footnotetext{
(a) Permanent address: Physics Department, State University of New York, Stony Brook, New York 11794. ${ }^{1} \mathrm{D}$. O. Wineland, R. Drullinger, and F. Walls, Phys. Rev. Lett. 40, 1639 (1978); W. Neuhauser et al., Phys. Rev. Lett. $\overline{41}, 233$ (1978).

${ }^{2}$ R. Frisch, Z. Phys. 86, 42 (1933).

${ }^{3}$ T. W. Hänsch and A. Schawlow, Opt. Commun. 13, 68 (1975).

${ }^{4}$ A. Ashkin, Phys. Rev. Lett. $\underline{40}, 729$ (1978); A. Ashkin and J. Gordon, Opt. Lett. 4, 161 (1979). Each of these has an excellent bibliography.

${ }^{5} \mathrm{R}$. Schieder, H. Walther, and L. Woste, Opt. Commun. $\underline{5}, 337$ (1972)。

${ }^{6}$ J. E. Bjorkholm et al., Phys. Rev. Lett. $\underline{41}, 1361$ (1978).

${ }^{7}$ V. S. Letokhov, V. G. Minogin, and B. D. Pavlik, Opt. Commun. 19, 72 (1976).

${ }^{8}$ V. I. Balykin, V. S. Letokhov, and V. I. Mishin, Zh. Eksp. Teor. Fiz. 78, 376 (1980) [Sov. Phys. JETP 51, 692 (1980)], and Pis'ma Zh. Teor. Fiz. 29, 614 (1979) [JETP Lett. 29, 614 (1979)].

${ }^{9}$ William D. Phillips, in Proceedings of the Seventh International Conference on Atomic Physics, Cambridge, Massachusetts, 1980 (unpublished).

${ }^{10} \mathrm{~V}$. G. Minogin, Opt. Commun. 34, 265 (1980)

${ }^{11} \mathrm{~J}$. Bjorkholm, R. Freeman, A. Ashkin, and D. Pearson, Opt. Lett. $\underline{5}, 111,210$ (E) (1980).

${ }^{12}$ Recent data taken with a new 1.1-m-long solenoid show peak $C$ at velocity less than $25 \%$ of $v_{0}$. It is much narrower and taller than that shown in Fig. 3 . This advance will be described in a later publication by the present authors and J. V. Prodan.
} 